\title{
An Architecture for Intelligent Systems Based on Smart Sensors
}

\author{
John Schmalzel, Senior Member, IEEE, Fernando Figueroa, Jon Morris, Student Member, IEEE, \\ Shreekanth Mandayam, Member, IEEE, and Robi Polikar, Member, IEEE
}

\begin{abstract}
Based on requirements for a next-generation rocket test facility, elements of a prototype intelligent rocket test facility (IRTF) have been implemented. The preliminary results provide the basis for future advanced development and validation using rocket test stand facilities at Stennis Space Center (SSC). Key components include distributed smart sensor elements integrated using a knowledge-driven environment. One of the specific goals is to imbue sensors with the intelligence needed to perform self-diagnosis of health and to participate in a hierarchy of health determination at sensor, process, and system levels. We have identified issues important to further development of health-enabled networks, which should be of interest to others working with smart sensors and intelligent health management systems.
\end{abstract}

Index Terms-Health management, IEEE 1451, integrated system health management (ISHM), integrated vehicle health monitoring (IVHM), rocket testing, sensor fusion, smart sensors.

\section{INTRODUCTION}

A LONG-TERM center goal at NASA's John C. Stennis Space Center (SSC) is the formulation and implementation of a framework for an intelligent rocket test facility (IRTF). ${ }^{1}$ The IRTF is to provide reliable, high-confidence measurements for a variety of propulsion test articles. Specific objectives include the following:

1) definition of a framework and architecture that supports implementation of highly autonomous methodologies founded on basic physical principles and embedded knowledge;

2) inclusion of smart sensors;

3) modeling of processes and other system elements;

4) development of appropriate communications protocols to enable complex interactions to support timely and highquality flow of information among the system elements;

5) development and validation of lab-scale prototypes of key system elements.

Though our application is next-generation rocket test facilities, other applications using this approach are much wider and include monitoring of shuttle launch operations, air and spacecraft

Manuscript received June 15, 2004; revised May 6, 2005.

J. Schmalzel, J. Morris, S. Mandayam, and R. Polikar are with the Electrical and Computer Engineering Department, Rowan University, Glassboro, NJ 08028 USA (e-mail: j.schmalzel@ieee.org).

F. Figueroa is with the Program Development Directorate, Technology Development and Transfer Office, Stennis Space Center, MS 39529 USA.

Digital Object Identifier 10.1109/TIM.2005.851477

${ }^{1}$ The opinions expressed herein are those of the authors and are not to be construed as those of NASA. operations and health monitoring, and other large-scale industrial system operations such as those found in processing and manufacturing plants.

Elements of a prototype IRTF have been implemented. An early objective was the development of distributed smart sensor elements in a knowledge-driven environment. Preliminary results provide the basis for advanced development and validation using rocket test stand facilities at SSC. We have identified issues important to further development of complex networks, which should be of interest to others working with smart sensors and intelligent health management systems.

Evolution of aerospace systems toward complex structures with distributed intelligence can improve performance, further safety, and enhance quality, while offering improved cost benefits. SSC has been actively pursuing ways to manage the complexity and improve the quality and cost of testing rocket engines. Engine test articles include the space shuttle main engine (SSME) and a range of engine development programs for future space flight. Using rocket engine testing as the model for autonomous systems makes sense for two reasons. 1) Rocket test facilities are essentially complete propulsion systems. Even when testing only components, the test facility also assumes the role of missing engine subsystems. Developing autonomous systems in support of ground-based testing will have direct application to flight propulsion systems [1]. 2) SSC is focused on delivering high-quality data to its propulsion test customers. Data must be accurate and have high integrity, while maintaining safe operation and providing timely services at reasonable costs. Autonomous system development that improves quality of data while improving safety and cost-effectiveness also has application to a wide spectrum of aerospace applications. Such techniques are also important to a broad range of commercial interests including nondestructive testing, power generation, manufacturing, military applications, chemical plants, and transportation systems.

Much work has been undertaken to develop standards for smart sensor communication, such as IEEE 1451 [2]. For example, Lee [3] describes the nature of one such sensor interface standard. Similarly, other investigators have reported on developing smart sensors and sensor interfaces. For example, Paschalidis [4], Hogenbirk et al. [5], and Ferrari et al. [6] describe the development of smart sensors. In particular, these and other reported smart sensors use a variety of common communication protocols such as $\mathrm{I}^{2} \mathrm{C}$, SPI, and Internet-based communication.

In our work, we have adopted a generalized model of the IEEE 1451 smart sensor as shown in Fig. 1. A transducer is supported by a smart transducer interface module (STIM), which 


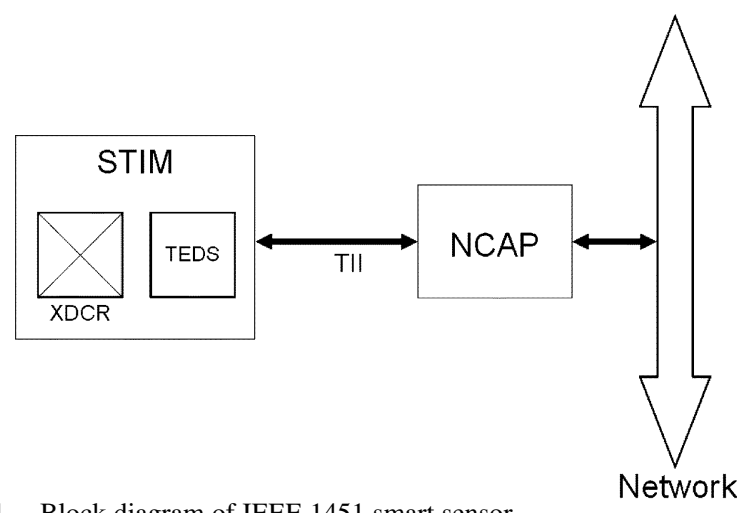

Fig. 1. Block diagram of IEEE 1451 smart sensor.

in turn communicates with a network-capable application processor (NCAP) over a transducer-independent interface (TII). For prototype smart sensors with integral STIM and NCAP, we have chosen to assign the functionality of the transducer electronic data sheet (TEDS) to the NCAP to simplify development time. Similarly, we have adopted variations of the other elements (STIM, TII, and NCAP) in our prototype system in order to provide near-term development and test flexibility. Future development will be redirected to transform our smart sensor architecture to be compliant with the IEEE 1451 standards.

Others have investigated frameworks for smart sensor and intelligent systems. For example, Guo et al. [7], working in the area of sensor fusion in robotic networks, developed an approach emphasizing communication between sensors to avoid high communication requirements with a central fusion center. In our approach, the IRTF is modeled as a rigorous hierarchy to postpone the issues of inter-sensor communication to a later date. While we like the idea of largely autonomous sensors, there are many issues of cooperation and control which need to be addressed. However, we can adopt their schema of smart sensor attributes including prediction, planning, updating, communication, and assimilation. In fact, sensor fusion is one area that we have identified as a core IRTF technology. This is because collections of sensors must be logically combined together into processes that involves fusing data from a potentially large number of sensors. Sensor fusion is of interest to a variety of application areas including robotics as described by Luo and Kay [8] and novel adaptive data fusion algorithms as developed by Polikar [9].

\section{APPROACH}

The earliest definition of our proposed architecture consists of three major elements as shown in Fig. 2. One or more shared networks allow all elements to cooperate in order to perform the intended system function, which in turn is composed of a collection of processes. Each process is made up of a collection of sensors, actuators, and other primitive components.

In order for this simplified approach to be meaningful, a number of knowledge elements must be linked. That is, each entity-sensor, process, and system-consists of a series of databases, which contain all pertinent information. In turn, these must be linked so that appropriate data can be exchanged and to support learning and adaptation as the system continues

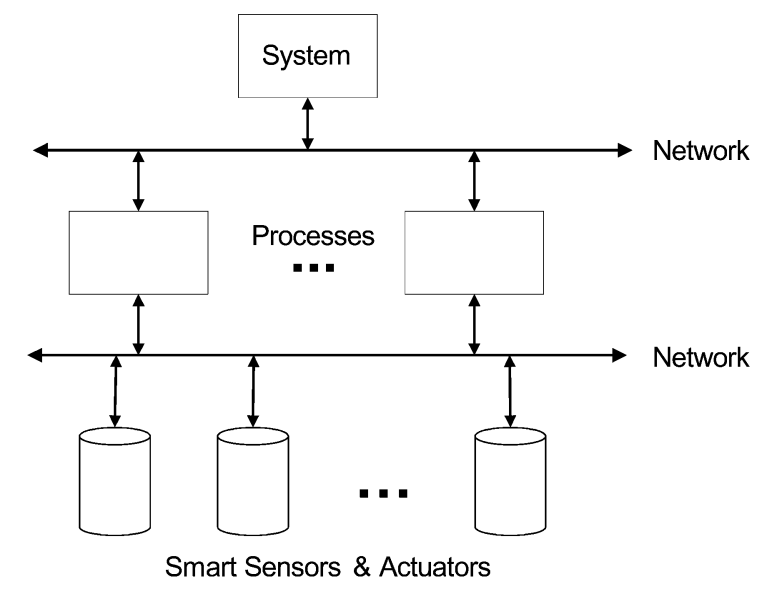

Fig. 2. Generalized block diagram of IRTF.

to evolve. For example, in the case of smart sensors, their associated database must contain the TEDS elements such as manufacturer, calibration coefficients, etc. In addition, there must also be components describing health conditions and metrics for each type of transducer and for the types of application environments. This aspect is considered further in a later section.

A second database would need to contain descriptions of the sensors that are associated with each process. Note that sensors and actuators can be shared between multiple processes-e.g., a flow sensor on a pipe between a storage tank and a test article could be both a member of the process controlling tank flow and the process controlling the infeed to the test article. Finally, a third database captures the higher level knowledge required to organize the information across the entire system. Fig. 3 elaborates the model emphasizing the knowledge bases that support each element of the hierarchy and the relationships between them. A key feature of the IRTF is the evaluation of condition for all elements performed both autonomously and using feedback from other higher order elements.

\section{DISCUSSION}

\section{A. Prototype IRTF}

The key component of our prototype IRTF is Gensym's G2 software, ${ }^{2}$ which is an expert system development environment designed to handle complex intelligent systems. G2 supports development of layered system behaviors analogous to the hierarchical autonomous architecture we seek to develop. We have developed a novel $\mathrm{G} 2$ framework that integrates system, processes, and smart sensors as shown in Fig. 4. An important attribute of the G2 development is the support for object-oriented components. Thus, common elements such as different smart sensors can be instantiated as a class and then new members inherit common attributes. This simplifies development and promises reuse by exchanging such models with other developers.

An important feature is the G2 gateway services that better support key performance issues. There are gateways for Internet-based smart sensors, file servers, and other application programs such as Matlab. ${ }^{3}$ In particular, many condition eval-

${ }^{2}$ [Online]. GenSym, Inc. Burlington, MA. Available: www.gensym.com

${ }^{3}$ [Online]. Mathworks, Inc., Natick, MA. Available: www.mathworks.com 


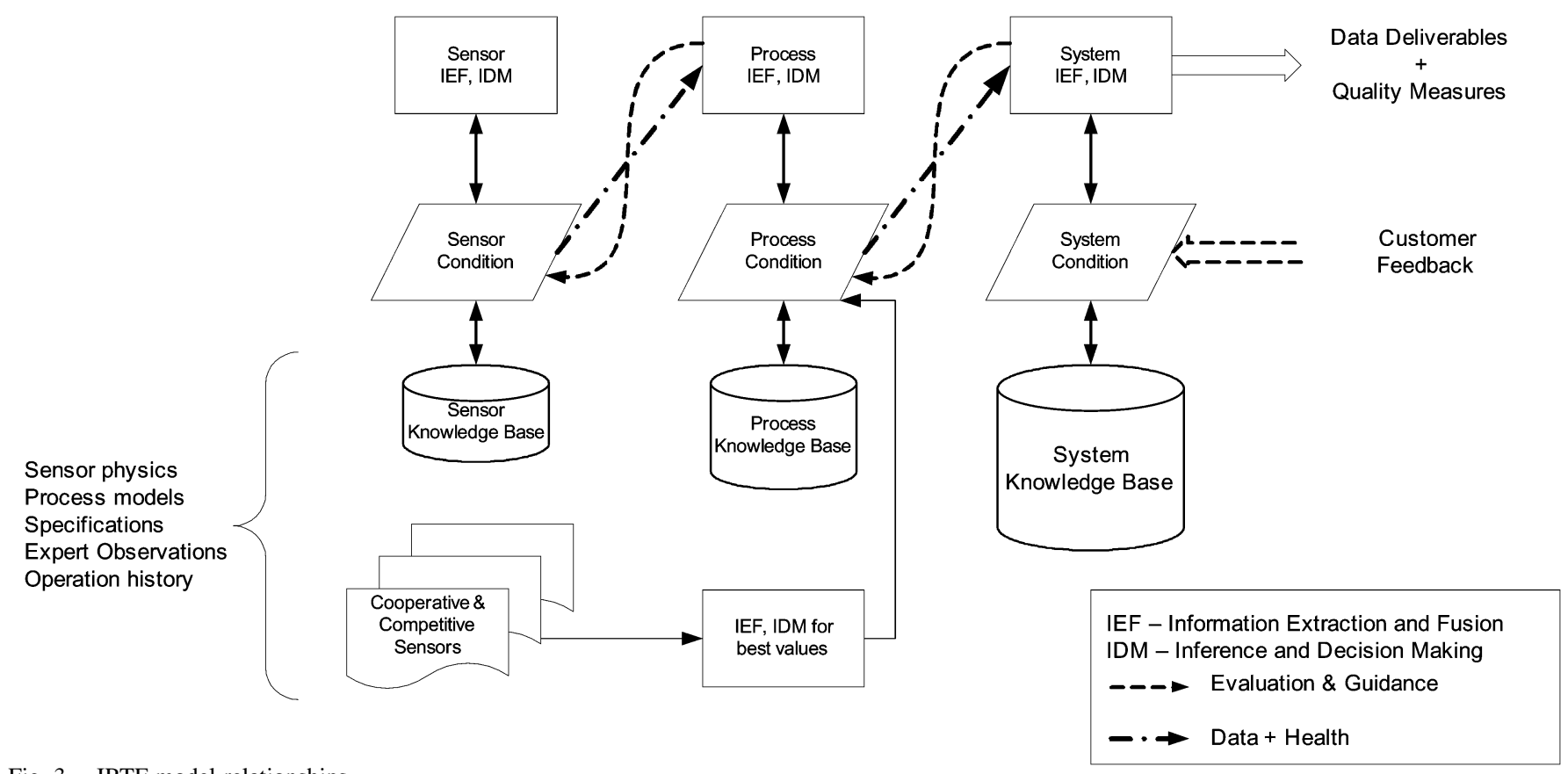

Fig. 3. IRTF model relationships.

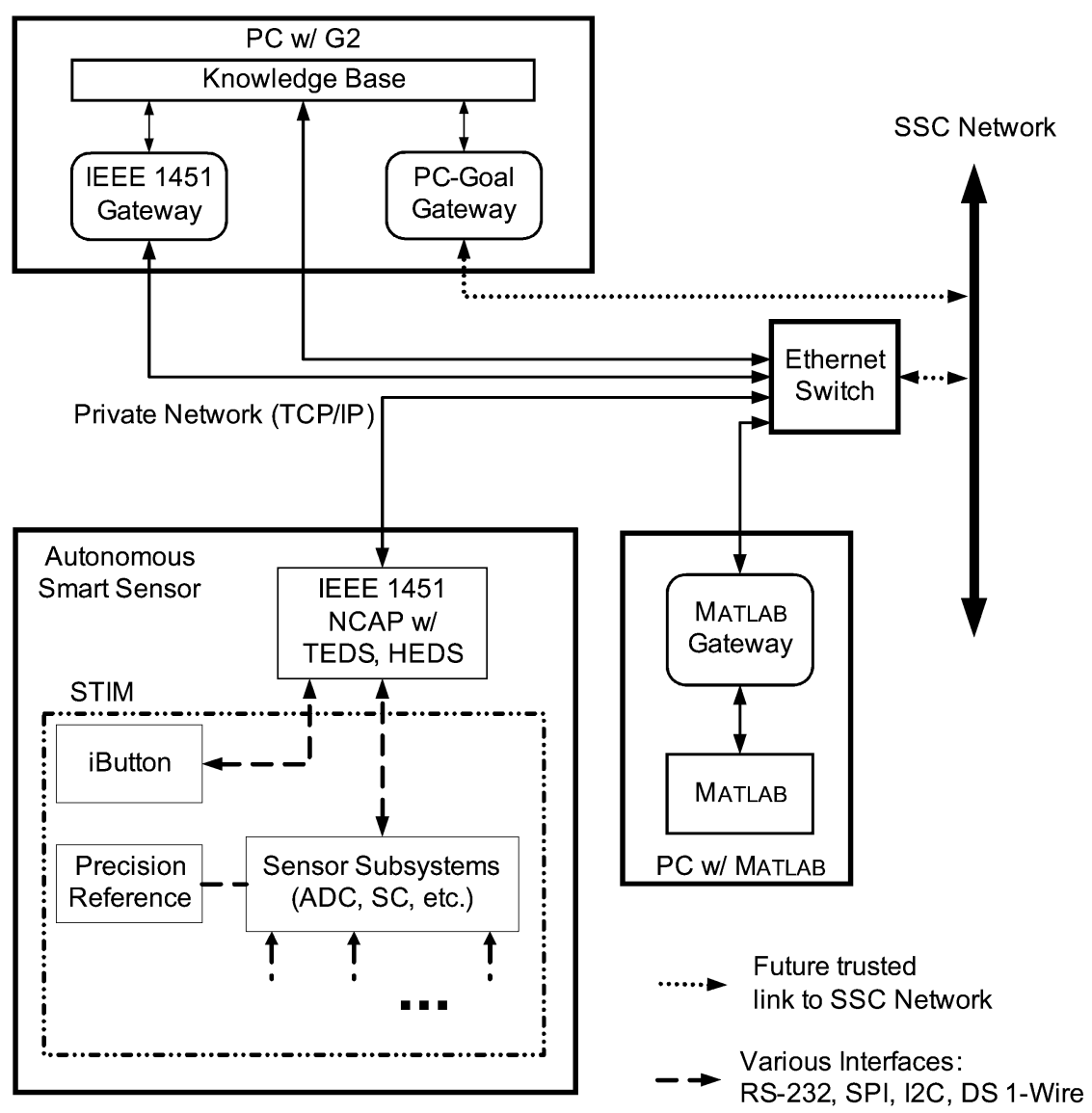

Fig. 4. Prototype IRTF.

uation algorithms involve large data structures and require complex analyses-e.g., fast Fourier transforms (FFT) and Wavelet transforms. Imposing these computational burdens on the G2 host results in throughput problems; we have addressed this problem by distributing certain analysis tasks to additional nodes.
Another result is the development of smart sensor cores based on an Ethernet core microcontroller ${ }^{4}$ with interfaces for $\mathrm{I}^{2} \mathrm{C}$, SPI, RS-232, and iButtons ${ }^{5}$ to support a spectrum of sensor types

${ }^{4}$ [Online]. Z-World, Davis, CA. Available: www.zworld.com

${ }^{5}$ [Online]. Maxim Integrated Products, Inc., Sunnyvale, CA. Available: www.maxim-ic.com 


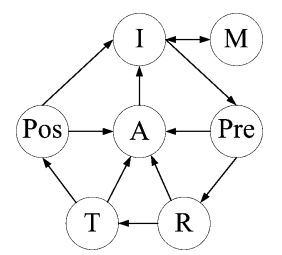

Fig. 5. System states: idle (I), maintenance (M), pretest (Pre), ready (R), test (T), abort (A), and post test (Pos).

TABLE I

LIST OF REPRESENTATIVE SENSOR ANOMALIES

\begin{tabular}{cc}
\hline Anomaly No. & $\begin{array}{c}\text { Behavior Noted on } \\
\text { Discrepancy Report }\end{array}$ \\
\hline 1 & Signal Limits (High, Low) \\
2 & Noise Limits (High, Low) \\
3 & Saturation (Pos, Neg) \\
4 & Bandwidth \\
5 & Noise Type (Impulsive, \\
& Continuous) \\
6 & Rise Time \\
7 & Fall Time \\
8 & Nominal Value \\
9 & Alias \\
10 & Impossible \\
11 & Other Instrumentation \\
& (Dead zone, Gain, Offset, \\
& Etc.) \\
\hline
\end{tabular}

and features. A novel addition to the smart sensor NCAP and TEDS functionality is the addition of health-related information at the sensor. This provides a means for tagging condition parameters onto the data stream. In aggregate, we term this the "health electronic data sheet" (HEDS), which is analogous to and an extension of TEDS.

\section{B. Health Condition Monitoring}

Sensor failure models needed to be developed. To accomplish this, we accessed the large body of sensor failures captured as part of routine discrepancy reports (DRs) that are triggered by any system anomaly. This very complete database represents many years of experience on every major test stand in the SSC inventory. Table I summarizes some of the common sensor failures that have been identified.

A second issue occurs because the health condition needs to be correlated to the state of the system. That is, any given fault condition is likely to be modified by the current state of the system. For example, the rise-time and fall-time parameters are strongly dependent on system state. A sample sensor variable can illustrate this point: If the system is in an "idle" state, it is likely to be in a long-term steady-state condition in which most measurement variables will experience very little change. Thus, a temperature variable would be expected to follow only environmental forcings and exhibit low rise and fall times. In contrast, during a pretest state, many cryogen lines would be undergoing chill down. In this state, the rise and fall times would be expected to be much faster than for the idle state. Fig. 5 shows the state transitions for a typical test stand. This requires that all fault conditions be further modified by the system state and the corresponding database constructed to reflect these associations

\section{Near-Term Development Objectives}

This work is proceeding according to a spiral development approach in which successive design cycles add new features and extend the capabilities implemented in the previous cycles. The near-term development objectives include the following.

- Refine knowledge bases appropriate for the three core elements of the architecture that include component specifications, behavioral models (analytic, empirical, qualitative, etc.), test requirements, expert observations, and facility operation history. Develop interfaces for accessing the knowledge bases and the means for efficiently updating them.

- Define condition states for all key elements and methods for performing the condition assessment algorithms. Apply new versions of the Learn++ algorithm [9] to impart dynamic learning behavior.

- Mature the smart sensor architecture. Some of the current features include 1) power over Ethernet [10], 2) communication using TCP/IP, and 3) embedded data acquisition using 24-b analog-to-digital converter.

- Modify an available portable rocket engine test stand to provide a physical test bed to validate the IRTF design and evaluate its response to well-defined faults.

\section{CONCLUSION}

Based on requirements for a next-generation rocket test facility, We have implemented elements of a prototype intelligent rocket test facility. The early results have established a basis for future advanced development and validation using the rocket test stand facilities at Stennis Space Center (SSC). Smart sensor components play key roles in providing the distributed intelligence needed to perform diagnosis of overall health. They also contribute to the hierarchy of health determination at process and system levels. Many of the issues we have identified as important to further development of health-enabled networks should be of interest to others working with smart sensors and integrated system health management (ISHM).

\section{REFERENCES}

[1] F. Figueroa, W. Solano, C. Thurman, and J. Schmalzel, "A future vision of data acquisition: Distributed sensing, processing, and health monitoring," in Proc. IMTC, Budapest, Hungary, May 2001, pp. 20-23.

[2] IEEE Standard for a Smart Transducer Interface for Sensors and Actuators-Network Capable Application Processor (NCAP) Information Model [Online]. Available: www.ieee.org

[3] K. Lee, "Sensor networking and interface standardization," in Proc. 18th IMTC, 2001, pp. 147-152.

[4] N. P. Paschalidis, "A smart sensor integrated circuit for NASA's new millennium spacecraft," in Proc. ICECS, 1999, pp. 1787-1790.

[5] E. J. Hogenbirk, H.-J. Verhoeven, and J. H. Huijsing, "An integrated smart sensor for flow and temperature with I2C bus interface: FTS2," in Proc. Int. Sym. Circuits Systems, 1995, pp. 2225-2228.

[6] P. Ferrari, A. Flammini, D. Marioli, and A. Taroni, "A low-cost internetenabled smart sensor," in Proc. Sensors, 2002, pp. 1549-1554.

[7] C.-H. Guo, X.-G. Wang, W.-H. Qian, and C.-X. Lin, "A new framework of distributed multisensor fusion intelligent systems," in Proc. Int. Conf. Ind. Tech., 1996, pp. 460-464.

[8] R. Luo and M. Kay, "Data fusion and sensor integration: State-of-the-Art 1990s," in Data Fusion in Robotics and Machine Intelligence, Abidi and Gonzales, Eds. Boston, MA: Academic, 1992, pp. 7-136. 
[9] R. Polikar, L. Udpa, S. S. Udpa, and V. Honavar, "Learn++: An incremental learning algorithm for multilayer perceptrons," in Proc. IEEE 25th Int. Conf. Acoustics, Speech, Signal Processing, vol. 6, 2000, pp. 3466-3469.

[10] IEEE Standard for Information Technology_-Part 3: Carrier Sense Multiple Access with Collision Detection (CSMA/CD) Access Method and Physical Layer Specifications Amendment: Data Terminal Equipment (DTE) Power via Media Dependent Interface (MDI) [Online]. Available: www.ieee.org

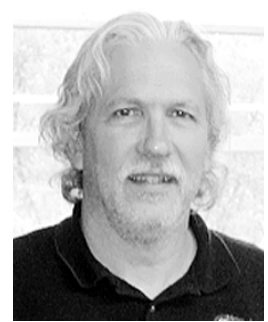

John Schmalzel (SM'00) received the B.S.E.E., M.S.E.E., and Ph.D. degrees in engineering from Kansas State University, Manhattan, in 1973, 1977, and 1980, respectively.

He is currently a Professor and Chair of the Department of Electrical and Computer Engineering, Rowan University, Glassboro, NJ. His research interests include smart sensors, intelligent systems for health monitoring, vehicular technologies, and biomedical devices. He spent much of 2003 working in the Test Technology Development and Commercialization group at Stennis Space Center, MS.

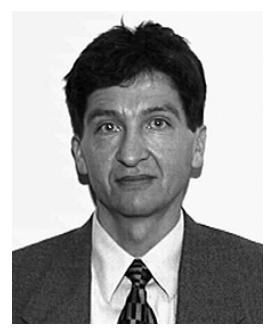

Fernando Figueroa received the B.S.M.E. degree from the University of Hartford, Hartford, CT, in 1983 and the M.S. and Ph.D. degrees in mechanical engineering from the Pennsylvania State University, State College, in 1984 and 1988, respectively.

$\mathrm{He}$ is currently with NASA's John C. Stennis Space Center, MS. His areas of interest include modeling methodologies to embed intelligence in systems and sensors, intelligent integrated system health management, instrumentation and sensors, ultrasonic sensing, fiber-optic sensors, robotics, automation, and controls. He is currently the Principal Investigator for a project to mature integrated system health management technologies.

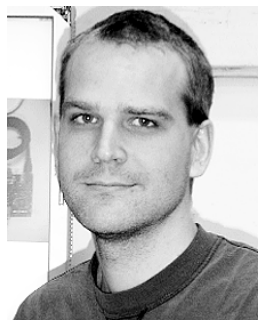

Jon Morris (S'99) received the B.S.E.C.E. degree from Rowan University, Glassboro, NJ, in 2003. He is currently pursuing the M.S. degree in engineering at Rowan University.

His interests include embedded system design, low-frequency instrumentation, intelligent system design and implementation based on $\mathrm{G} 2$, and smart sensors.

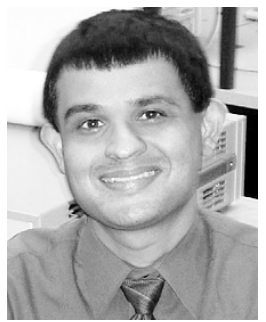

Shreekanth Mandayam (M'96) received the B.E. degree in electronics engineering from Bangalore University, Bangalore, India, in 1990 and the M.S. and Ph.D. degrees in electrical engineering (communications and signal processing) from Iowa State University, Ames, in 1993 and 1996, respectively.

$\mathrm{He}$ is an Associate Professor of Electrical and Computer Engineering at Rowan University, Glassboro, NJ. His research interests include multisensor data fusion, imaging and image processing, advanced visualization, and artificial neural networks applied to nondestructive evaluation. He directs the Imaging and Nondestructive Evaluation Laboratory at Rowan University.

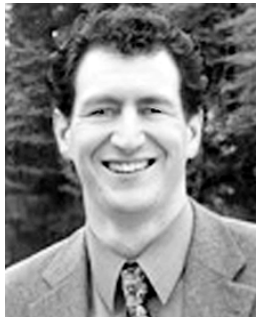

Robi Polikar (M'00) received the B.S. degree in electronics and communications engineering from Istanbul Technical University, Istanbul, Turkey, in 1993 and the M.S. and Ph.D. degrees, both co-majors in biomedical engineering and electrical engineering, from Iowa State University, Ames, in 1995 and 2000, respectively.

$\mathrm{He}$ is currently an Assistant Professor with the Department of Electrical and Computer Engineering at Rowan University, Glassboro, NJ. His current research interests include signal processing, pattern recognition, neural systems, machine learning, and computational models of learning, with applications to biomedical engineering and imaging, chemical sensing, nondestructive evaluation, and testing. 